\title{
Galactic axions search with a superconducting resonant cavity
}

D. Alesini, ${ }^{1}$ C. Braggio, ${ }^{2,3}$ G. Carugno, ${ }^{2,3}$ N. Crescini, ${ }^{4,3}$ D. D’Agostino, ${ }^{5}$ D. Di Gioacchino, ${ }^{1}$ R. Di Vora, ${ }^{2, *}$ P. Falferi, ${ }^{6,7}$ S. Gallo, ${ }^{2,3}$ U. Gambardella, ${ }^{5}$ C. Gatti, ${ }^{1,}$ G. Iannone, ${ }^{5}$ G. Lamanna, ${ }^{8}$ C. Ligi, ${ }^{1}$ A. Lombardi, ${ }^{4}$ R. Mezzena, ${ }^{7,9}$ A. Ortolan, ${ }^{4}$ R. Pengo, ${ }^{4}$ N. Pompeo, ${ }^{10}$ A. Rettaroli, ${ }^{1,11,}$ G. Ruoso, ${ }^{4}$ E. Silva, ${ }^{10}$ C. C. Speake, ${ }^{12}$ L. Taffarello, ${ }^{2}$ and S. Tocci ${ }^{1}$

${ }^{1}$ INFN, Laboratori Nazionali di Frascati, Frascati, Roma, Italy

${ }^{2}$ INFN, Sezione di Padova, Padova, Italy

${ }^{3}$ Dipartimento di Fisica e Astronomia, Padova, Italy

${ }^{4}$ INFN, Laboratori Nazionali di Legnaro, Legnaro, Padova, Italy

${ }^{5}$ Dipartimento di Fisica E.R. Caianiello, Fisciano, Salerno, Italy and INFN, Sez. di Napoli, Napoli, Italy

${ }^{6}$ Istituto di Fotonica e Nanotecnologie, CNR Fondazione Bruno Kessler, I-38123 Povo, Trento, Italy

${ }^{7}$ INFN, TIFPA, Povo, Trento, Italy

${ }^{8}$ Dipartimento di Fisica and INFN, Sezione di Pisa, Pisa, Italy

${ }^{9}$ Dipartimento di Fisica, Povo, Trento, Italy

${ }^{10}$ Department of Engineering, University Roma Tre, Rome, Italy

${ }^{11}$ Dipartimento di Matematica e Fisica Università di Roma 3, Roma, Italy

${ }^{12}$ School of Physics and Astronomy, University of Birmingham, Birmingham, United Kingdom

(Received 18 March 2019; published 1 May 2019)

To account for the dark-matter content in our Universe, postinflationary scenarios predict for the QCD axion a mass in the range $\left(10-10^{3}\right) \mu \mathrm{eV}$. Searches with haloscope experiments in this mass range require the monitoring of resonant cavity modes with frequency above $5 \mathrm{GHz}$, where several experimental limitations occur due to linear amplifiers, small volumes, and low quality factors of copper resonant cavities. In this paper, we deal with the last issue, presenting the result of a search for galactic axions using a haloscope based on a $36 \mathrm{~cm}^{3} \mathrm{NbTi}$ superconducting cavity. The cavity worked at $T=4 \mathrm{~K}$ in a $2 \mathrm{~T}$ magnetic field and exhibited a quality factor $Q_{0}=4.5 \times 10^{5}$ for the TM010 mode at $9 \mathrm{GHz}$. With such values of $Q$, the axion signal is significantly increased with respect to copper cavity haloscopes. Operating this setup, we set the limit $g_{a \gamma \gamma}<1.03 \times 10^{-12} \mathrm{GeV}^{-1}$ on the axion photon coupling for a mass of about $37 \mu \mathrm{eV}$. A comprehensive study of the $\mathrm{NbTi}$ cavity at different magnetic fields, temperatures, and frequencies is also presented.

DOI: 10.1103/PhysRevD.99.101101

\section{INTRODUCTION}

The axion is a pseudoscalar particle predicted by $\mathrm{S}$. Weinberg [1] and F. Wilczek [2] as a consequence of the mechanism introduced by R. D. Peccei and H. Quinn [3] to solve the "strong CP problem." Axions are also wellmotivated dark-matter (DM) candidates with expected mass laying in a broad range from pico-electron-volts to few milli-electron-volts [4]. Postinflationary scenarios restrict this range to $\left(10-10^{3}\right) \mu \mathrm{eV}$ [4], where a rich experimental program will probe the axion existence in the next decade. Among the experiments, ADMX [5], HAYSTAC [6], ORGAN [7], CULTASK [8], and KLASH [9] will use a haloscope, i.e., a detector composed of a resonant cavity immersed in a strong magnetic field as proposed by P. Sikivie [10]. When the resonant frequency of the cavity $\nu_{c}$ is tuned to the corresponding axion mass

\footnotetext{
divora@pd.infn.it

claudio.gatti@lnf.infn.it

*alessio.rettaroli@lnf.infn.it
}

$m_{a} c^{2} / h$, the expected power deposited by DM axions is given by [6]

$P_{a}=\left(g_{\gamma}^{2} \frac{\alpha^{2}}{\pi^{2}} \frac{\hbar^{3} c^{3} \rho_{a}}{\Lambda^{4}}\right) \times\left(\frac{\beta}{1+\beta} \omega_{c} \frac{1}{\mu_{0}} B_{0}^{2} V C_{m n l} Q_{L}\right)$,

where $\rho_{a}=0.45 \mathrm{GeV} / \mathrm{cm}^{3}$ is the local DM density, $\alpha$ is the fine-structure constant, $\Lambda=78 \mathrm{MeV}$ is a scale parameter related to hadronic physics, and $g_{\gamma}$ is a model-dependent parameter equal to $-0.97(0.36)$ in the Kim-Shifman-Vainshtein-Zakharov (KSVZ) (DineFischler-Srednicki-Zhitnitskii) axion model [11,12]. It is related to the coupling appearing in the Lagrangian $g_{a \gamma \gamma}=$ $\left(g_{\gamma} \alpha / \pi \Lambda^{2}\right) m_{a}$. The second set of parentheses contains the vacuum permeability $\mu_{0}$, the magnetic field strength $B_{0}$, the cavity volume $V$, its angular frequency $\omega_{c}=2 \pi \nu_{c}$, the coupling between the cavity and receiver $\beta$, and the loaded quality factor $Q_{L}=Q_{0} /(1+\beta)$, where $Q_{0}$ is the unloaded quality factor; here, $C_{m n l} \simeq O(1)$ is a geometrical factor depending on the cavity mode. 
The axion mass range studied by haloscopes up to now is limited to a few micro-electron-volts. Exploring larger ranges at higher values requires the excitation of modes with frequency above a few gigahertz where several experimental limitations occur:

(i) The technology of linear amplifier limits the sensitivity [13].

(ii) Conversion volumes are smaller since the normal modes resonant frequencies are inversely proportional to the cavity radius.

(iii) the anomalous skin effect reduces the copper cavities quality factor at high frequencies.

Solutions to the first two issues are proposed for instance in Refs. [14,15], respectively.

The optimum value of the $Q$-factor for haloscopes is approximately $10^{6}$, as estimated by the coherence time of DM axions [16]. A $10 \mathrm{GHz}$ copper cavity, cooled at cryogenic temperature, barely reaches $Q \sim 10^{5}$, a value that rapidly decreases with increasing frequency. In this paper, we present a substantial improvement obtained for the quality factor with a "superconducting haloscope" composed of a superconducting cavity (SCC) operated in high magnetic fields. This activity has been done within the QUAX experiment, which searches DM axions using a ferromagnetic haloscope [16,17]. The same experimental apparatus can be used as a Sikivie haloscope [10] exploiting the TM010 mode of the cylindrical cavity. In this work, we substitute the copper cavity with a superconducting one, to increase the quality factor and thus the measurement sensitivity. We refer to the Primakoff haloscope of the QUAX Collaboration as "QUAX-ar."

In Sec. II, we describe the characterization of the SCC and the measurement setup, while in Sec. III, we present the results of the axion search done by operating the SCC in magnetic field and the corresponding exclusion limit on the coupling $g_{a \gamma \gamma}$. Finally, in Sec. IV, we discuss the future prospects of the QUAX-ar experiment for the Primakoff axion search.

\section{EXPERIMENTAL APPARATUS}

\section{A. Superconducting cavity}

To increase the quality factor and match the optimal condition for the coupling to cosmological axions, it is natural to consider SCCs as they were widely studied in accelerator physics. However, in axion searches, these are operated in strong magnetic fields that, on the one hand, weaken superconductivity and, on the other, are screened by the superconducting material. To overcome both these limitations, we designed a cavity divided in two halves, each composed by a type II superconducting body and copper end caps. Type II superconductors are in fact known to have a reduced sensitivity to the applied magnetic field. Moreover, magnetic field penetration in the inner cavity volume may be facilitated by interrupting the screening

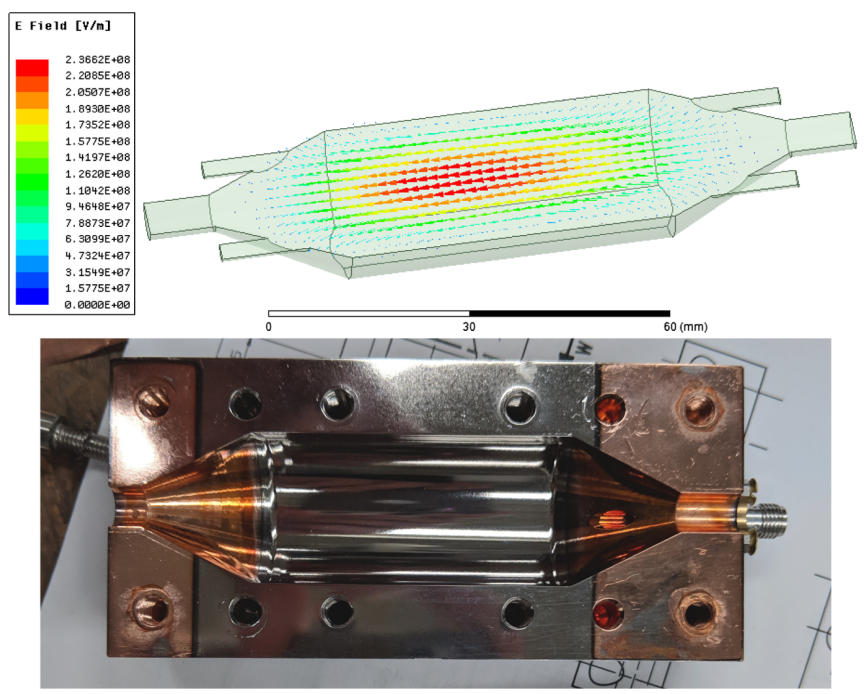

FIG. 1. The upper image represents the electric field of $9.08 \mathrm{GHz}$ TM010 mode in arbitary amplitude units, while the lower photo is one of the two halves of the superconducting cavity.

supercurrents with the insertion of a thin $(30 \mu \mathrm{m})$ copper layer between the two halves.

The cavity layout is shown in the upper part of Fig. 1, featuring two identical copper semicells with cylindrical body and conical end caps to reduce current dissipation at interfaces. The cylindrical body is $50 \mathrm{~mm}$ long with diameter $26.1 \mathrm{~mm}$, while the cones are $19.5 \mathrm{~mm}$ long. For the detection of axions through its interaction with the electron spin [17], part of the cylinder was flattened to break the angular symmetry and prevent the degeneration of mode TM110. A finite element calculation performed with ANSYS HFSS [18] of the TM010 mode used for Primakoff axion detection gives a frequency $\nu_{c}^{\text {sim }}=$ $9.08 \mathrm{GHz}$ and a coefficient $C_{m n l}=0.589$ in a volume $V=36.43 \mathrm{~cm}^{3}$. The calculated field profile of this mode is shown in Fig. 1. Because of this hybrid geometry, the quality factor is expressed as

$$
\frac{1}{Q_{0}}=\frac{R_{s}^{\mathrm{cyl}}}{G_{\mathrm{cyl}}}+\frac{R_{S}^{\mathrm{cones}}}{G_{\mathrm{cones}}}
$$

where $R_{s}$ are the surface resistances. The simulation yields $G_{\text {cones }}=6270.11 \Omega$ and $G_{\text {cyl }}=482.10 \Omega$. At $9 \mathrm{GHz}$ and $4 \mathrm{~K}$ temperature, the surface resistance for copper is $R_{s}^{\mathrm{Cu}}=$ $4.9 \mathrm{~m} \Omega$ [19]. A pure copper cavity with this geometry would have $Q_{0}^{\mathrm{Cu}} \simeq 9 \times 10^{4}$, while an hybrid cavity with copper cones and no losses on the cylindrical surface would have $Q_{0}^{\max }=G_{\text {cones }} / R_{s}^{\mathrm{Cu}} \simeq 1.3 \times 10^{6}$.

To test these promising simulation results, a prototype of the cavity was fabricated as shown in the lower part of Fig. 1. The inner cylindrical part of the cavity was coated by means of a $10 \mathrm{~cm}$ planar magnetron equipped with a 
$\mathrm{NbTi}$ target. The estimated coating thickness ranges between 3 to $4 \mu \mathrm{m}$. To minimize the losses due to the interaction of fluxons [20] with the superconducting microwave current, only the cylindrical body, where the currents of the mode TM010 are parallel to the applied field, were coated, as evidenced by the different colors of the lower picture of Fig. 1.

We characterized the cavity in a thermally controlled gasflow cryostat equipped with an $8 \mathrm{~T}$ superconducting magnet located at Laboratori Nazionali di Frascati. No copper layer was inserted between the two halves. Two tunable antennas were coupled to the cavity mode and connected through coaxial cables to a vector network analyzer for the measurement of the reflection and transmission waveforms, $S_{11}(\nu)$ and $S_{12}(\nu)$. The unloaded quality factor $Q_{0}$ was extracted from a simultaneous fit of the two waveforms. An expected systematic error of $\pm 5 \%$ follows from the fit procedure. At $4.2 \mathrm{~K}$ and no applied external magnetic field, we measured $Q_{0}=1.2 \times 10^{6}$, in agreement with the maximal expected value $Q_{0}^{\max }$ and corresponding to a surface resistance of the $\mathrm{NbTi} R_{s}^{\mathrm{NbTi}}=(20 \pm 20) \mu \Omega$. We repeated the measurement for different values of the applied magnetic field in the range $0-5 \mathrm{~T}$ both in zero-field cooling (ZFC) and field cooling (FC), thus reducing the temperature of the cavity without or with external magnetic field,

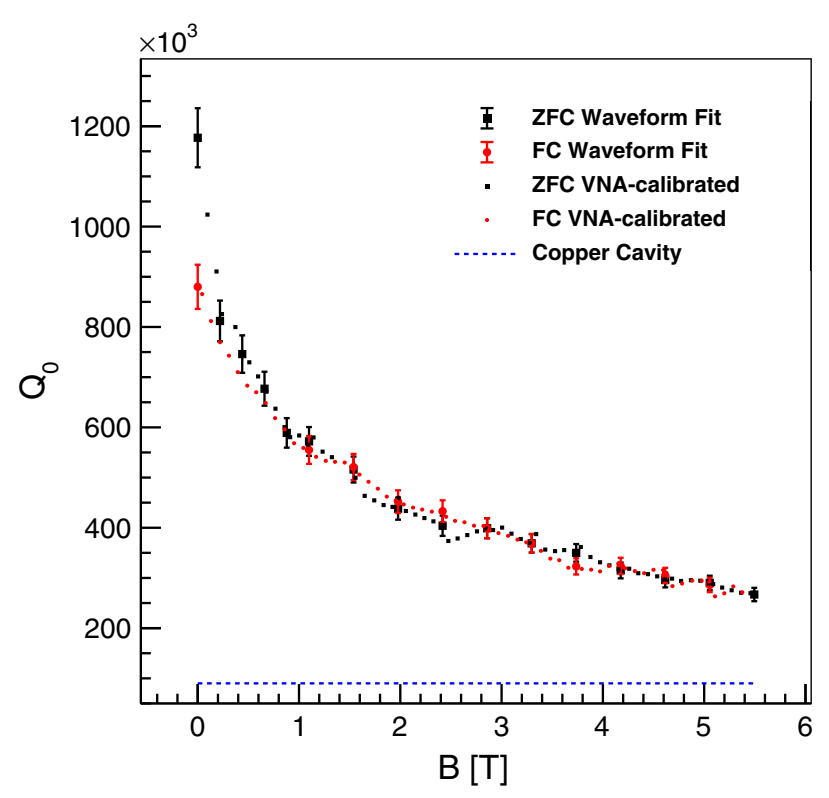

FIG. 2. Unloaded quality factor $Q_{0}$ vs external magnetic field amplitude $B$ for the hybrid copper-NbTi cavity, compared with the one of a copper cavity (blue horizontal line). ZFC points are measured by cooling the cavity before increasing the field; the opposite procedure was used for FC data (see the text for details). Unloaded quality factors $Q_{0}$ indicated by large markers with error bars were derived from fits to the reflection and transmission waveforms, $S_{11}(\nu)$ and $S_{12}(\nu)$. These measurements were used to correct the loaded quality factors $Q_{L}$ measured by the vector network analyzer, shown, after the correction, as small markers without error bars. respectively. The results are shown in Fig. 2. For $B=2 \mathrm{~T}$, the nominal field used in our axion search, we measured $Q_{0}^{2 \mathrm{~T}}=4.5 \times 10^{5}$, a factor of approximately 5 better than a bulk copper cavity; at $5 \mathrm{~T}$ we measured $Q_{0}^{5 \mathrm{~T}}=2.95 \times 10^{5}$, a factor of approximately 3.3 better than a copper cavity. Comparing FC and ZFC measurements, we observe a systematic difference below about $0.5 \mathrm{~T}$ due to magnetic field trapping in the superconducting material. For higher values, the measurements agree within the errors, showing that the magnetic field is able to penetrate the cavity walls and that the superconductor is in the flux flow state [21].

In a recent analysis [22], the variation of the surface resistance of this cavity with the magnetic field was interpreted by taking into account the vortex-motion contribution within the Gittleman and Rosenblum (GR) model $[23,24]$. In particular, the depinning frequency [23] was estimated to be about $44 \mathrm{GHz}$. Below this frequency, losses due to vortex motion are suppressed, while they are maximal at higher frequency where the flux-flow resistivity reaches the value $\rho_{\mathrm{ff}}=c_{\mathrm{ff}} \rho_{n} B / B_{c 2}$. Here, $B$ is the applied dc field, $c_{\mathrm{ff}}=0.044$ is a correction taking into account mutual orientation of fluxons and microwave currents, $\rho_{n}=7.0 \times 10^{-7} \Omega \mathrm{m}$ is NbTi resistivity in the normal state, and $B_{c 2}=13 \mathrm{~T} \times\left(1-\left(T / T_{c}\right)^{2}\right)$ is the temperature-dependent critical field [22]. With this set of parameters, we computed, according to the GR model, the surface resistance of NbTi for different values of the applied dc field and of the cavity-mode frequency. Comparing it with the anomalous surface-resistance of copper [19], we estimated the crossing frequency for which the losses in $\mathrm{NbTi}$ are equal to the losses in copper. The result is shown in Fig. 3. At $T=4.2 \mathrm{~K}$, the $\mathrm{NbTi}$ cavity is expected to show lower losses up to an applied field of about 4.5 T. For higher fields, the crossing frequency rapidly decreases down to

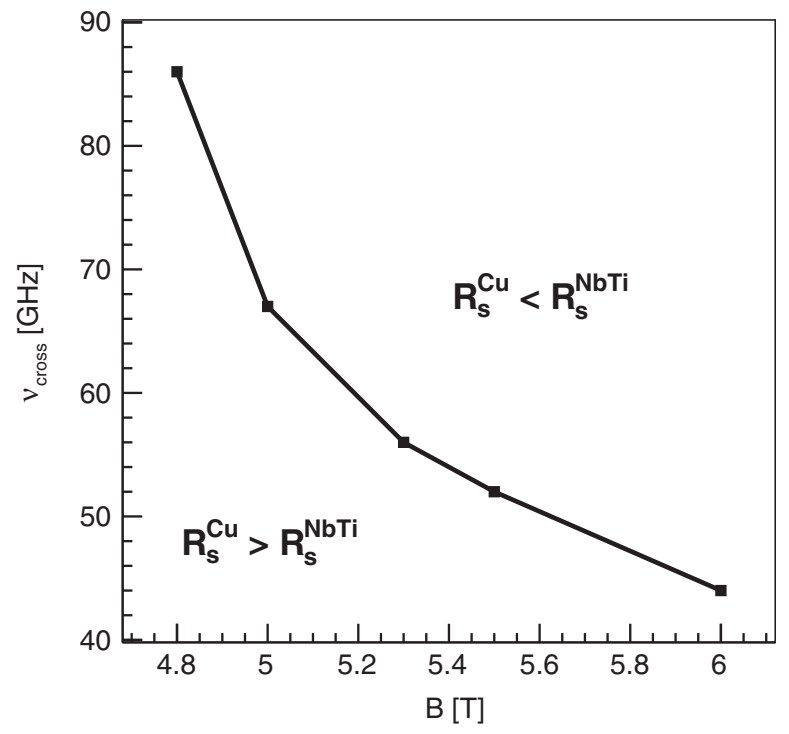

FIG. 3. Estimated crossing frequency as a function of applied $B$ field. 
$45 \mathrm{GHz}$ for $B=6 \mathrm{~T}$. By lowering the temperature down to $100 \mathrm{mK}$, we expect a $20 \%$ improvement of the $Q_{0}$ and larger values for the crossing frequency. In fact, at this temperature, the critical field $B_{c 2}$ reaches its maximum value, $13 \mathrm{~T}$, reducing the flux-flow resistivity.

\section{B. Magnet and readout electronics}

A replica of the cavity described in Sec. II A was mounted in the experimental site at Laboratori Nazionali di Legnaro (LNL), which hosts an apparatus capable of searching for galactic axions [17]. The scheme of the apparatus is shown in Fig. 4. The SCC is inside a vacuum chamber inserted in a superconducting magnet. The magnet is a $\mathrm{NbTi}$ compensated solenoid, $15 \mathrm{~cm}$ bore and $50 \mathrm{~cm}$ height, generating a central field of $2 \mathrm{~T}$ with homogeneity better than $20 \mathrm{ppm}$ on a $20-\mathrm{mm}$-long line along the central axis. A superconducting switch is installed to perform measurements also in persistent current mode. The bias current of $50 \mathrm{~A}$ is supplied by a high-stability current generator. The magnet and the vacuum chamber are immersed in a LHe bath at the temperature of $4.2 \mathrm{~K}$. The cavity is instrumented with two antennas. A weakly coupled one is used to inject probe signals in the cavity with

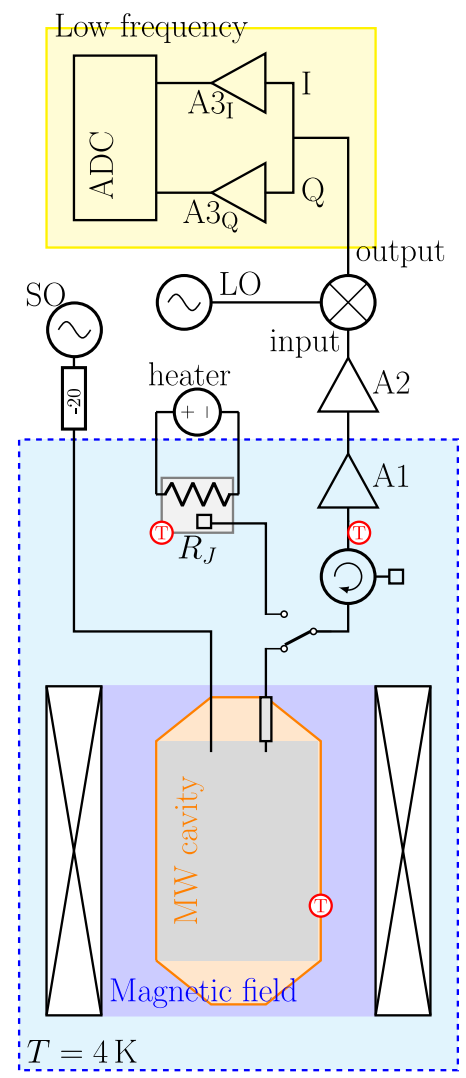

FIG. 4. QUAX-ar setup: the blue dashed line encloses the liquid helium temperature part of the apparatus, the yellow rectangle stands for the low-frequency electronics, and the red circled $T$ s represent the thermometers. See the text for further details. a source oscillator. The second antenna, with a variable coupling, is connected through a coaxial cable to a low noise cryogenic high electron mobility transistor amplifier (A1) with gain $G_{1} \simeq 40 \mathrm{~dB}$. Before reaching the amplifier, the coaxial cable is connected to a cryogenic switch and then to an isolator. The switch, used for calibration purposes, allows the replacement of the cavity output with the output of a resistor $\left(R_{J}\right)$. The temperature of the resistor is kept constant by a heater and read by a thermometer. The setup is completed by a second field effect transistor amplifier (A2) at room temperature with gain $G_{2} \simeq 39 \mathrm{~dB}$ and a down-converter mixer referenced to a local oscillator (LO). The in-phase (I) and quadrature (Q) components of the mixer output are further magnified by two identical room temperature amplifiers $\left(A 3_{I, Q}\right)$ with $G_{3} \simeq 50 \mathrm{~dB}$ each and acquired by a 16 bit analog to digital converter (ADC) sampling at $2 \mathrm{MHz}$. The acquisition program controls both the oscillators, the ADC and the applied magnetic field. Three thermometers monitor the temperature of the cavity, of the resistance $R_{J}$ and of the amplifier A1, with typical temperatures of $T_{c}=4.3 \mathrm{~K}, T_{J}=4.5 \mathrm{~K}$, and $T_{a}=5.1 \mathrm{~K}$, respectively.

\section{EXPERIMENTAL RESULTS}

With the LNL setup described in Sec. II, we performed the first search for galactic axions using a SCC. The frequency of the TM010 cavity mode, $\nu_{c}=9.07 \mathrm{GHz}$, was in good agreement with the simulated value $\nu_{c}^{\text {sim }}=9.08 \mathrm{GHz}$. A copper layer was used to interrupt circular screening supercurrents and allow magnetic field penetration. No impact was observed on the quality factor, since the longitudinal microwave currents of the TM010 mode were unaffected by the interposed mask. The penetration of the magnetic field in the cavity volume was verified by means of a $1 \mathrm{~mm}$ yttrium iron garnet sphere positioned on the cavity axis with a teflon holder. Hybridization of the sphere ferromagnetic resonance with the TM110 mode at $14 \mathrm{GHz}$ occurs with a field of $0.5 \mathrm{~T}$, showing a typical double resonance curve of which the Lorentzian peaks have line widths equal to the average of the yttrium iron garnet and cavity line widths. The observation of this effect confirmed that the field distortions were at the level of $100 \mathrm{ppm}$ or lower over the sphere volume. Measurements in the dispersive regime, i.e., with ferromagnetic resonance frequency different from the cavity one, also gave similar results.

To down-convert and acquire the signal, the frequency of the local oscillator fed to the mixer was set to $\nu_{\mathrm{LO}}=$ $\nu_{c}-500 \mathrm{kHz}$, and the $I$ and $Q$ components were combined to extract the right part of the down-converted spectra, where the cavity resonance lies. We determined the total gain $G$ and the noise temperature $T_{n}$ of the amplification chain by heating the resistor $R_{J}$ from about 4.5 to $8.5 \mathrm{~K}$ and by measuring the temperature and the corresponding Johnson noise [25]. The resulting values are 


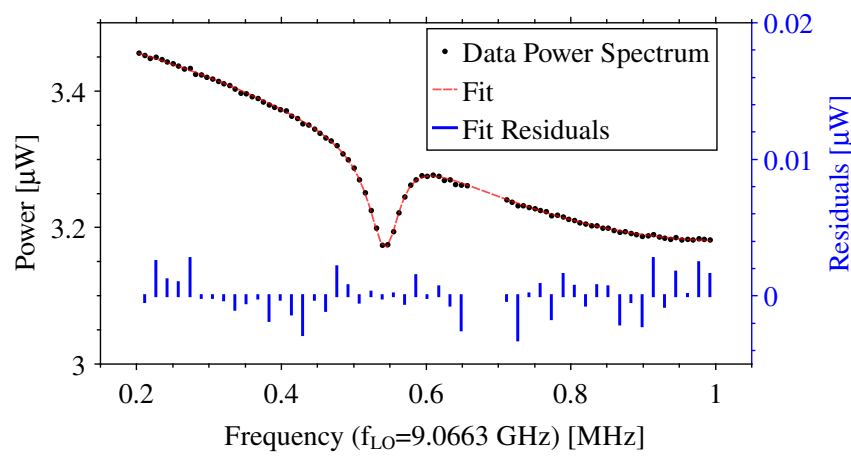

FIG. 5. Down-converted rf power at the ADC input. The collected data are the black dots (the errors are within the symbol dimension), and the red-dashed line is the fit with the residuals reported in blue. A part of the bandwidth was removed due to systematic disturbances.

$T_{n}=(11.0 \pm 0.1) \mathrm{K}, \quad G=(1.96 \pm 0.01) \times 10^{12}$.

We set the magnetic field to $2 \mathrm{~T}$ and measured the cavity quality factor $Q_{0}=4.02 \times 10^{5}$, compatible within the errors with our previous measurement. Finally, we critically coupled the tunable antenna and measured the loaded quality factor $Q_{L}=2.01 \times 10^{5}$. With high quality factors, the temperature stability of the system is a critical issue; in fact, we observed a drift of the cavity resonance frequency of the order of the line width in the timescale of an hour. Thus, the integration time was limited to $\Delta t=20 \mathrm{~min}$. The collected data were analyzed with a fast Fourier transform (FFT) with a resolution bandwidth of $\Delta \nu=7812.5 \mathrm{~Hz}$ close to the axion line width, to maximize the signal-tonoise ratio (SNR) in a single bin. The resulting 9375000 FFTs were rms averaged, and the bins with disturbances introduced by the low-frequency electronics were excluded from the analysis. The power spectrum was fit using a degree 5 polynomial to account for the off-resonance part of the spectra, whose variation is due to the nonuniform gain of ADC and amplifiers. We modeled the on resonance spectrum with the absorption profile of a Lorentzian curve. Since the temperature of the cavity was lower than the one of the isolator, the spectrum of power reflected by the cavity shows a minimum at the resonance frequency. The measured spectrum, together with the fit and residuals, is shown in Fig. 5. The residuals are distributed according to a Gaussian probability density function, and their standard deviation scales as $\sqrt{\Delta t}$ as expected. To get the equivalent power at the cavity output, we divide the power measured at the ADC input by the total measured gain $G$ in Eq. (3). Its standard deviation is $\sigma_{P}=6.19 \times 10^{-22} \mathrm{~W}$. This value is compatible with the prediction of the Dicke relation [26]

$$
\sigma_{P}=k_{B} T_{S} \sqrt{\frac{\Delta \nu}{\Delta t}} \simeq 5.5 \times 10^{-22} \mathrm{~W}
$$

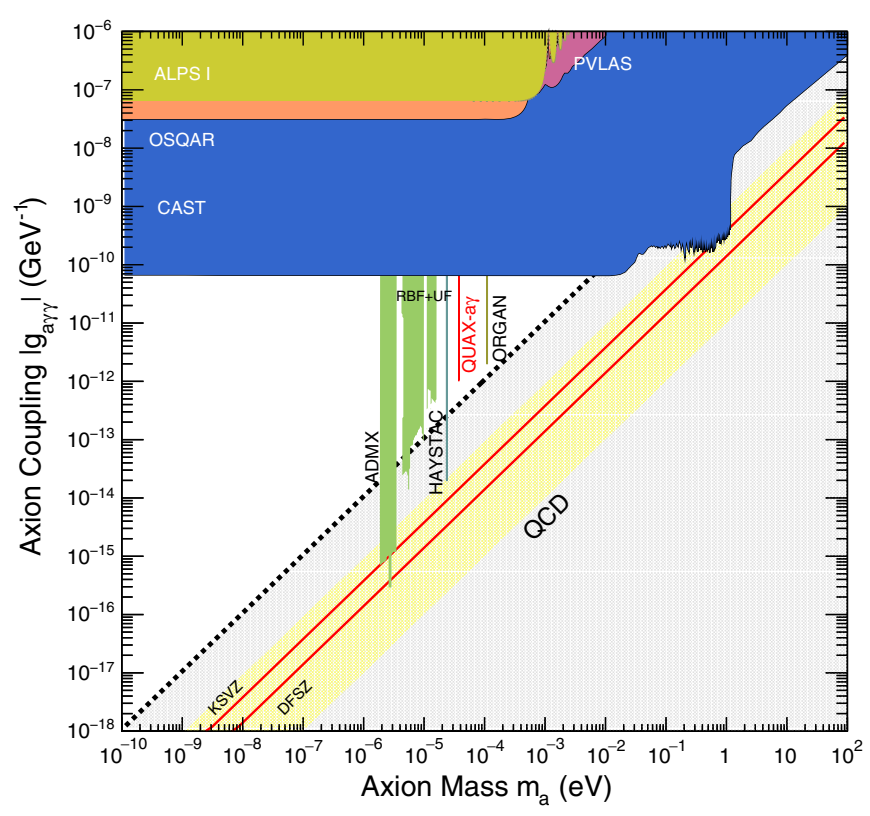

FIG. 6. Exclusion plot of the axion-photon coupling. The red lines with a yellow error band show the theoretical predictions for the KSVZ and Dine-Fischler-Srednicki-Zhitnitskii axions $[11,12]$. The grey area shows the prediction form other hadronic axions models [27]. The experimental limits, shown by the different color regions, are obtained with light shining through a wall experiments [28,29], from changes in laser polarization [30] helioscopes [31] and haloscopes [5-7,32,33], as the one in the present work.

where $T_{S}=T_{n}+T_{c}=15.3 \mathrm{~K}$. The expected power generated by KSVZ axions in our cavity, derived from Eq. (1), is

$$
\begin{aligned}
P_{\mathrm{a}} & =1.85 \times 10^{-25} \mathrm{~W}\left(\frac{V}{0.0361}\right)\left(\frac{B}{2 \mathrm{~T}}\right)^{2}\left(\frac{g_{\gamma}}{-0.97}\right)^{2} \\
& \times\left(\frac{C}{0.589}\right)\left(\frac{\rho_{a}}{0.45 \mathrm{GeV} \mathrm{cm}^{-3}}\right)\left(\frac{\nu_{c}}{9.067 \mathrm{GHz}}\right)\left(\frac{Q_{L}}{201000}\right) .
\end{aligned}
$$

The $95 \%$ single-sided confidence limit $(1.64 \sigma)$, shown in Fig. 6 , is $g_{\text {ary }}<1.03 \times 10^{-12} \mathrm{GeV}^{-1}$ in a frequency band of $45 \mathrm{kHz}$ at $\nu_{c}$ corresponding to a mass range of approximately $0.2 \mathrm{neV}$ around $m_{a} \simeq 37.5 \mu \mathrm{eV}$.

\section{CONCLUSIONS}

SCCs appear as a natural choice for high-frequency haloscopes as their quality factor matches the one of cosmological axions. In this work, we characterized a SCC by testing it under a high magnetic field at cryogenic temperature. After successful tests, we performed a singlemass axion search, extracting an upper limit on $g_{a \gamma \gamma}$ for a narrow frequency band. This result is limited by the low 
magnetic field, the high system temperature, and the small cavity volume. A new experimental setup is now in preparation and consists of a dilution refrigerator, a quantum limited Josephson parametric amplifier (JPA), and an $8 \mathrm{~T}$ superconducting magnet. At $50 \mathrm{mK}$ with a quantum limited amplifier, such as a JPA, the system temperature is reduced to about $400 \mathrm{mK}$. Operating a $20-\mathrm{cm}-$ long NbTi cavity in a $5 \mathrm{~T}$ magnetic field, we expect, from our measurements, a quality factor $Q_{0}^{5 \mathrm{~T}, 50 \mathrm{mK}}=2.95 \times 1.2 \times 10^{5}=3.54 \times 10^{5}$, a factor 4 better than a copper cavity. With this setup, the expected 95\% exclusion limit would be $g_{a \gamma \gamma}<4 \times 10^{-14} \mathrm{GeV}^{-1}$ for $m_{a} \simeq 37.5 \mu \mathrm{eV}$ a value that touches the region expected for KSVZ axions.

\section{ACKNOWLEDGMENTS}

We would like to thank M. Iannarelli and G. Pileggi for their support in the preparation of the Laboratori Nazionali di Frascati setup and F. Tabacchioni and M. Martini for their help with the cryogenic system. We also thank E. Berto, F. Calaon, M. Tessaro, M. Zago, and M. Rebeschini for their work on the mechanics and electronics of the LNL setup; L. Castellani and G. Galet for building the magnet current source; and N. Toniolo, M. Gulmini, and S. Marchini for the help with the data acquisition system. Finally, we would like to thank A. Benato for mechanical construction of the resonant cavities and C. Pira for the deposition of the NbTi film.
[1] S. Weinberg, Phys. Rev. Lett. 40, 223 (1978).

[2] F. Wilczek, Phys. Rev. Lett. 40, 279 (1978).

[3] R. D. Peccei and H. R. Quinn, Phys. Rev. Lett. 38, 1440 (1977); Phys. Rev. D 16, 1791 (1977).

[4] M. Tanabashi et al. (Particle Data Group), Phys. Rev. D 98, 030001 (2018).

[5] S. Asztalos, E. Daw, H. Peng, L. J. Rosenberg, C. Hagmann, D. Kinion, W. Stoeffl, K. vanBibber, P. Sikivie et al., Phys. Rev. D 64, 092003 (2001); S. J. Asztalos et al., Phys. Rev. Lett. 104, 041301 (2010).

[6] S. Al Kenany et al., Nucl. Instrum. Methods Phys. Res., Sect. A 854, 11 (2017); B. M. Brubaker, L. Zhong, Y. V. Gurevich, S. B. Cahn, S. K. Lamoreaux, M. Simanovskaia, J. R. Root, S. M. Lewis, S. Al Kenany et al., Phys. Rev. Lett. 118, 061302 (2017).

[7] B. T. McAllister, G. Flower, E. N. Ivanov, M. Goryachev, J. Bourhill, and M. E. Tobar, Phys. Dark Universe 18, 67 (2017).

[8] W. Chung, Proc. Sci., CORFU2015 (2016) 047.

[9] D. Alesini, D. Babusci, D. Di Gioacchino, C. Gatti, G. Lamanna, and C. Ligi, arXiv:1707.06010; C. Gatti et al., arXiv: 1811.06754.

[10] P. Sikivie, Phys. Rev. Lett. 51, 1415 (1983); Phys. Rev. D 32, 2988 (1985).

[11] J. Kim, Phys. Rev. Lett. 43, 103 (1979); M. A. Shifman, A. I. Vainshtein, and V. I. Zakharov, Nucl. Phys. B166, 493 (1980).

[12] M. Dine, W. Fischler, and M. Srednicki, Phys. Lett. B 104B, 199 (1981); A. R. Zhitnitsky, Sov. J. Nucl. Phys. 31, 260 (1980) [Yad. Fiz. 31, 497 (1980)].

[13] S. K. Lamoreaux, K. A. van Bibber, K. W. Lehnert, and G. Carosi, Phys. Rev. D 88, 035020 (2013).

[14] L. S. Kuzmin, A. S. Sobolev, C. Gatti, D. Di Gioacchino, N. Crescini, A. Gordeeva, and E. Ilichev, IEEE Trans. Appl. Supercond. 28, 2400505 (2018).

[15] J. Jeong, S. W. Youn, S. Ahn, J. E. Kim, and Y. K. Semertzidis, Phys. Lett. B 777, 412 (2018).

[16] R. Barbieri, C. Braggio, G. Carugno, C. S. Gallo, A. Lombardi, A. Ortolan, R. Pengo, G. Ruoso, and C. C. Speake, Phys. Dark Universe 15, 135 (2017).
[17] N. Crescini et al., Eur. Phys. J. C 78, 703 (2018).

[18] https://www.ansys.com/-products/-electronics/-ansys-hfss.

[19] G. E. H. Reuter and E. H. Sondheimer, Proc. R. Soc. A 195, 336 (1948).

[20] A. A. Abrikosov, J. Phys. Chem. Solids 2, 199 (1957).

[21] J. Bardeen and M. J. Stephen, Phys. Rev. 140, A1197 (1965).

[22] D. Di Gioacchino et al., IEEE Trans. Appl. Supercond. 29, 3500605 (2019).

[23] J. I. Gittleman and B. Rosenblum, Phys. Rev. Lett. 16, 734 (1966).

[24] N. Pompeo and E. Silva, Phys. Rev. B 78, 094503 (2008).

[25] C. Braggio, G. Bressi, G. Carugno, F. Della Valle, G. Galeazzi, and G. Ruoso, Nucl. Instrum. Methods Phys. Res., Sect. A 603, 451 (2009).

[26] R. H. Dicke, Rev. Sci. Instrum. 17, 268 (1946).

[27] L. Di Luzio, F. Mescia, and E. Nardi, Phys. Rev. Lett. 118, 031801 (2017).

[28] K. Ehret et al. (ALPS Collaboration), Phys. Lett. B 689, 149 (2010).

[29] R. Ballou, G. Deferne, M. Finger, M. Finger, L. Flekova, J. Hosek, S. Kunc, K. Macuchova, K. A. Meissner et al. (OSQAR Collaboration), Phys. Rev. D 92, 092002 (2015).

[30] F. Della Valle A. Ejlli, U. Gastaldi, G. Messineo, E. Milotti, R. Pengo, G. Ruoso, and G. Zavattini (PVLAS Collaboration), Eur. Phys. J. C 76, 24 (2016).

[31] M. Arik et al. (CAST Collaboration), Phys. Rev. D 92, 021101 (2015); V. Anastassopoulos et al. (CAST Collaboration), Nat. Phys. 13, 584 (2017).

[32] S. DePanfilis, A.C. Melissinos, B. E. Moskowitz, J. T. Rogers, Y. K. Semertzidis, W. U. Wuensch, H. J. Halama, A. G. Prodell, W. B. Fowler, and F. A. Nezrick, Phys. Rev. Lett. 59, 839 (1987); W. Wuensch, S. De PanfilisWuensch, Y. K. Semertzidis, J. T. Rogers, A. C. Melissinos, H. J. Halama, B. E. Moskowitz, A. G. Prodell, W. B. Fowler, and F. A. Nezrick, Phys. Rev. D 40, 3153 (1989).

[33] C. Hagmann, P. Sikivie, N. S. Sullivan, and D. B. Tanner, Phys. Rev. D 42, 1297 (1990). 